\title{
Ernst Mach et le principe d'économie de pensée : de l'évolutionnisme à la méthodologie normative
}

Sabine Plaud

\section{OpenEdition}

1 Journals

Édition électronique

URL : https://journals.openedition.org/cps/2108

DOI : $10.4000 /$ cps. 2108

ISSN : 2648-6334

Éditeur

Presses universitaires de Strasbourg

Édition imprimée

Date de publication : 15 décembre 2012

Pagination : 307-324

ISBN : 978-2-354100-51-3

ISSN : $1254-5740$

Référence électronique

Sabine Plaud, «Ernst Mach et le principe d'économie de pensée : de l'évolutionnisme à la

méthodologie normative », Les Cahiers philosophiques de Strasbourg [En ligne], 32 | 2012, mis en ligne le

15 mai 2019, consulté le 09 novembre 2022. URL : http://journals.openedition.org/cps/2108 ; DOI

https://doi.org/10.4000/cps.2108

\section{c) (i) (3)}

Creative Commons - Attribution - Pas d'Utilisation Commerciale - Partage dans les Mêmes Conditions 4.0 International - CC BY-NC-SA 4.0

https://creativecommons.org/licenses/by-nc-sa/4.0/ 


\section{Ernst Mach et le principe d'économie de pensée: de l'évolutionnisme à la méthodologie normative}

\section{Sabine Plaud}

La seconde moitié du $\mathrm{XX}^{\mathrm{e}}$ siècle a vu prospérer toute une génération de "physiciens-philosophes». Ces penseurs, tout en produisant des travaux intenses et innovants en matière de physique ou de mécanique, ne manquaient pas d'associer à ces recherches une démarche réflexive interrogeant la portée des découvertes opérées par les sciences de la nature, la signification de l'histoire de la physique, voire la nature de la connaissance en général. Ernst Mach (1838-1916) est à cet égard particulièrement représentatif de l'esprit de son temps. Auteur de très importantes contributions en physique (notamment sur les vitesses supersoniques) et en psychophysiologie, il a accompagné ses travaux d'une abondante réflexion sur l'histoire des sciences d'une part, sur l'origine et la nature des connaissances d'autre part. Nous n'aurons guère besoin de mentionner sa monumentale histoire de la mécanique de 1883, ouvrage dans lequel l'histoire des idées scientifiques est mise au service d'une enquête critique sur les principes de la science. Mais l'épistémologie de Mach s'expose également dans d'autres ouvrages: l'Analyse des sensations (1886), où l'enquête physiologique sur les données de la perception s'assortit d'un ensemble de thèses relatives à la nature du monde et à l'objectivité de la connaissance, les Leçons scientifiques populaires (Populär-wissenschaftliche Vorlesungen, 1896), les Principes de la théorie de la chaleur (Die Principien der Wärmelehre, 1896); enfin, La Connaissance et l'erreur (1905), texte tardif dans lequel Mach récapitule dans le détail les tenants de son épistémologie.

Or l'une des singularités de ce dernier texte est que, peut-être davantage qu'ailleurs, Mach y appuie sa théorie de la connaissance sur 
les acquis de la doctrine évolutionniste telle qu'elle fut proposée par Charles Darwin et Herbert Spencer. En effet, la doctrine évolutionniste trouve ici une articulation naturelle avec un principe qui court comme un fil conducteur tout au long de l'épistémologie machienne, à savoir le principe d'économie de pensée. Ce principe est bien connu: tel qu'il est formulé dans la Mécanique, il affirme que la logique de la science tient en "un problème de minimum qui consiste à exposer les faits aussi parfaitement que possible avec la moindre dépense intellectuelle $»^{1}$. Notons bien que le lexique économique n'est ici nullement métaphorique. Selon Mach, c'est au sens propre que la science est un commerce: il s'agit d'un commerce avec la nature, qui joue un rôle clé dans les activités humaines, et qui doit permettre aux hommes de prospérer au sein de leur environnement. Dans ses propres termes:

«Et pourquoi la science devrait-elle avoir honte d'un tel principe? Car la science elle-même n'est jamais qu'un - commerce [Geschäft]! Elle se donne bien pour tâche, avec le moindre travail possible, dans le temps le plus bref possible et même avec le moins de pensées possibles, d'acquérir autant de vérité éternelle et infinie qu'il est possible $»^{2}$.

On comprend alors aisément quel dialogue peut s'établir entre cette idée d'une logique économique de la science et la doctrine évolutionniste: le processus par lequel l'homme cherche à connaître le monde n'est en fait, selon Mach, que l'une des figures que peuvent recevoir les stratégies évolutives du vivant, de sorte que la connaissance du monde s'inscrit en continuité avec les procédures par lesquelles un vivant (en l'occurrence, l'homme) en vient à s'adapter progressivement à son environnement. C'est pourtant ce rapport apparemment évident entre économie de pensée et doctrine évolutionniste que nous souhaitons interroger ici, en nous demandant si, et comment, la pensée évolutionniste peut servir à fonder une authentique logique pour la science. Certes, l'idée de fournir

$1 C f$. Mach E., La Mécanique, Exposé historique et critique de son développement, Paris, Hermann, 1904, reproduit chez J. Gabay, Sceaux, 1987 [ci-après: M], p. 457: «Mais à cause de la courte durée de la vie et des limites resserrées de l'intelligence humaine, un savoir digne de ce nom ne peut être acquis que par la plus grande économie mentale. La science elle-même peut donc être considérée comme un problème de minimum, qui consiste à exposer les faits aussi parfaitement que possible, avec la moindre dépense intellectuelle».

2 Mach E., Populär-wissenschaftliche Vorlesungen, $3^{\mathrm{e}}$ édition, Leipzig, J. A. Barth, 1903 [ci-après: $P W V$ ], p. 16 [nous traduisons]. 
un compte rendu évolutionniste de la connaissance était déjà présente chez les représentants de l'évolutionnisme proprement dit, notamment chez Herbert Spencer dont les Principles of Psychology se proposent de fournir un exposé des procédures logiques, cognitives et scientifiques dans les termes de la doctrine darwinienne. Simplement, la perspective qui était alors adoptée restait principalement descriptive, n'ayant ni la prétention ni la capacité à se transformer en doctrine normative relative à la logique de la connaissance. En ce sens, l'idée même d'une «logique évolutionniste de la science» apparaissait pour le moins fragile. Mach, en revanche, maintient assurément le projet de proposer une explication psychologique des procédés scientifiques, comme en témoigne le soustitre apposé à La Connaissance et l'erreur: «Esquisses d'une psychologie de la recherche scientifique». Mais il n'entend pas moins proposer, sur cette base, une authentique méthodologie pour la recherche, et donc énoncer effectivement, à travers son principe d'économie de pensée, un ensemble de règles normatives, une logique pour la recherche scientifique.

Dans cet exposé, notre objectif sera donc de montrer comment Mach parvient (s'il y parvient) à transformer en logique normative de la science une théorie de la connaissance évolutionniste qui, dans son fondement, était essentiellement génétique et descriptive. Nous commencerons pour cela par présenter la théorie évolutionniste de la connaissance telle qu'elle est proposée par Spencer; puis nous examinerons la façon dont cette épistémologie évolutionniste est réinvestie par Mach à travers son principe d'économie de pensée, de façon à constituer une authentique méthodologie ou logique de la science.

\section{Spencer et la théorie évolutionniste de la connaissance}

Il n'est pas rare que l'on parle d'une épistémologie ou d'une logique évolutionniste. À cet égard, nous mentionnerons par exemple l'ouvrage de Paul Regnaud datant de 1897 et intitulé Précis de logique évolutionniste, où il s'agit principalement d'exploiter les ressources d'une linguistique évolutionniste pour comprendre les rapports de l'entendement avec le langage, ainsi que la logique qui naît de ces rapports. Plus généralement, l'idée principielle de la prétendue "logique évolutionniste» serait de décrire les opérations de l'entendement pour autant qu'elles s'enracinent dans des procédures d'adaptation du sujet à son environnement. Or on peut faire remonter l'un des points d'origine de ces tentatives dans 
les Principles of Psychology d'Herbert Spencer (1855), texte où il s'agit de rendre compte de la psychologie sur une base physiologique, et de naturaliser ainsi la connaissance dont les principes et la genèse doivent pouvoir être expliqués sur une base strictement biologique. En d'autres termes, l'ouvrage se présente comme une tentative de fournir une psychologie évolutionniste de la connaissance et du raisonnement. À cet égard, nous citerons Spencer lui-même quant à la façon dont on peut combler le hiatus apparent entre logique et psychologie:

«On pourrait affirmer qu'il ne relève pas de la province de la Logique que d'expliciter le fonctionnement de l'intellect - que celle-ci s'occupe de l'aspect objectif du raisonnement, et non de son aspect subjectif, qui concerne la Psychologie [...]. Et cela est vrai. Mais il semble y avoir une impression indéfinie et cependant générale selon laquelle une certaine vérité abstraite censée être impliquée dans tout syllogisme est ce que l'esprit reconnaît à chaque fois qu'il parcourt un syllogisme; et que la reconnaissance de cette vérité abstraite sous chaque incarnation particulière est le véritable acte de raisonnement $»^{3}$.

En d'autres termes, il n'est aux yeux de Spencer pas contradictoire de vouloir faire collaborer logique objective et psychologie subjective du raisonnement, en examinant la façon dont l'esprit parvient à reconnaître une vérité au cours de ses procédures logiques. Or cette articulation entre psychologie et logique que Spencer propose dans l'ouvrage se déploie en deux moments principaux: une partie analytique, qui s'emploie à décrire les procédures effectives du raisonnement humain ${ }^{4}$, tels que le syllogisme, la classification ${ }^{5}$ ou la dénomination ${ }^{6}$; et une partie synthétique ${ }^{7}$, où il s'agit non plus de décrire ou d'analyser les procédures logiques humaines, mais de les comprendre globalement et de les réinscrire dans leur ancrage évolutionniste. Nous citerons ici la façon dont Spencer ménage la transition vers cette partie synthétique:

3 Spencer H., The Principles of Psychology, Londres, Longman, Brown, Green and Longmans, 1855 [ci-après, $P P$ ], $\$ 38$, p. 95-96 [nous traduisons].

4 Voir la définition générale du raisonnement, id., $\$ 41$, p. 103: «tout acte de raisonnement est l'établissement d'une relation définie entre deux relations définies».

5 Cf. ibid., p. 104.

$6 \quad$ Ibid., $\$ 43$, p. 106.

7 Qui correspond à la Partie III de l'ouvrage, "General Synthesis», les deux premières parties étant consacrées à l'analyse générale et spéciale. 
"Si l'on se souvient que les lois de structure et de fonction doivent nécessairement s'harmoniser; et que la structure et les fonctions du système nerveux doivent se conformer aux lois de structure et de fonction en général [...] on verra que les généralisations ultimes de la Psychologie et de la Physiologie doivent être, telles qu'elles apparaissent ici, différentes faces de la même vérité primordiale. On verra qu'elles sont toutes deux des expressions du même principe fondamental de Vie»"

Ainsi, l'idée générale de Spencer est que les procédures cognitives et logiques de l'homme sont enracinées dans les lois de la vie, et qu'elles ne se dissocient que tardivement des manifestations primitives de cette dernière. Comme il le remarque lui-même:

«Bien que nous considérions communément la vie mentale et corporelle comme distinctes, il suffit de s'élever quelque peu au-dessus du point de vue ordinaire pour voir que celles-ci ne sont que des subdivisions de la vie en général; et qu'aucune ligne de démarcation ne peut être tirée entre elles, sinon arbitrairement ${ }^{9}$.

Il en résulte que la stratégie adoptée par la psychologie de la connaissance pour comprendre les lois du raisonnement sera tout simplement d'examiner les lois fondamentales de la vie dont le raisonnement est un dérivé, attendu que la vie en général est définie par Spencer comme «une combinaison définie de changements hétérogènes, à la fois simultanés et successifs » ${ }^{10}$. La thèse de Spencer est alors que la vie intellectuelle ou l'intelligence se différencient progressivement des phénomènes de la vie en général par leur tendance à s'ordonner exclusivement en séries $^{11}$, par exemple la succession des étapes du raisonnement dans le

8 PP, $\$ 105$, p. 202.

9 Id., $\$ 111$, p. 209 . Voir également ibid., $\$ 112$, p. 210 : «La vie corporelle et la vie mentale étant ainsi des divisions de la vie en général - étant liées l'une à l'autre comme des espèces dont la vie en général est le genre -, il résulte de la conclusion atteinte au dernier chapitre que la meilleure façon de trouver une vraie généralisation des phénomènes mentaux sera de les comparer aux phénomènes vitaux inférieurs, et de se demander quelles caractéristiques ces deux classes ont en commun".

10 Ibid., $\$ 115$, p. 217.

11 Cf. ibid., $\$ 172$, p. 301: "Concernant la nature de l'intelligence, nous parvenons donc à la conclusion qu'elle consiste en un certain ordre de changements, qui se distinguent de cet ordre inférieur de changements constituant la vie corporelle par cette particularité qu'au lieu d'être à la fois simultanés et successifs, ils sont successifs uniquement». 
syllogisme; et ce sont justement ces lois de succession que cherchera à élucider la psychologie de la connaissance dans son enquête sur les lois de l'intelligence ${ }^{12}$. Mentionnons ici le début du chapitre intitulé «La loi de l'intelligence»:

«Toute vie, qu'elle soit physique ou psychique, étant la combinaison de changements en correspondance avec des coexistences et séquences externes, il en résulte que si les changements qui constituent la vie psychique, ou l'intelligence, se produisent dans une succession, la loi de leur succession doit être la loi de leur correspondance ${ }^{13}$.

Simplement, on comprend que cette tâche qui incombe à la psychologie de la connaissance reste simplement descriptive: l'objectif de Spencer n'est pas ici de proposer une quelconque méthodologie normative pour la science, ni même de spécifier des principes qui permettraient d'accroître cette intelligence en améliorant la coordination entre réalité et représentation par des principes d'économie ou de simplicité. Les Principles of Psychology consacrent certes des pages à la croissance de l'intelligence ${ }^{14}$ : mais ces analyses demeurent génétiques, et étudient en fait les lois d'évolution qui, d'une espèce à l'autre, ont rendu possibles un affinement et un ajustement des procédures cognitives. Or à présent, nous souhaitons examiner la façon dont Mach, dans sa propre épistémologie fondée sur le principe d'économie de pensée, franchit un pas pour fonder, sur une base évolutionniste, quelque chose comme une authentique logique de la science.

\section{Ernst Mach : le principe d'économie de pensée comme méthode pour la science}

\section{Mach et les théories évolutionnistes de la connaissance}

Que Mach ait été inspiré par les théories évolutionnistes, c'est là quelque chose qui ne fait aucun doute, et qui est tout à fait assumé par lui dans ses différents textes. C'est ainsi que, dans sa conférence intitulée

12 Plus exactement, son objectif sera de découvrir non seulement les lois de succession au sein de la représentation, mais les lois qui garantissent la correspondance entre l'ordre interne des représentations et l'ordre externe des phénomènes qui leur correspondent.

13 Ibid., $\$ 173$, p. 303.

14 Voir la partie III, chapitre III : «The Growth of Intelligence». 
"Über Umbildung und Anpassung im naturwissenschaftlichen Denken" («Sur l'organisation et l'adaptation dans la pensée scientifique»), Mach formule la remarque suivante:

«Ainsi, la transformation des pensées telle que nous l'avons examinée nous apparaît comme une partie du développement général de la vie, de l'adaptation à un cercle d'action croissant ${ }^{15}$.

En cela, donc, Mach ne craint pas de proposer un compte rendu finalisé des procédures cognitives, voire de l'organisation physiologique humaine ${ }^{16}$, qu'il considère comme le résultat de stratégies d'adaptation. Plus exactement, la notion d'adaptation intervient à deux niveaux dans la théorie machienne de la connaissance: pour expliquer l'origine de la connaissance humaine, et pour rendre compte du rapport des pensées entre elles. Quant au premier point, on soulignera que, chez Mach, la pensée scientifique (et même la connaissance vulgaire) tirent leur origine d'un processus d'adaptation de l'homme à son environnement. En effet, on peut les interpréter comme l'un des moyens les plus sophistiqués que le vivant a pu mettre en œuvre pour réagir correctement à son environnement, notamment en prédisant les événements à venir en vue d'y adapter son action. À cet égard, mentionnons ce passage de l'Analyse des sensations qui est tout à fait explicite quant aux finalités pragmatiques qui s'attachent à la science en tant que stratégie d'adaptation:

"C'est par l'effort vers une adaptation avantageuse aux conditions de vie, et non à travers la connaissance pure et complète comme finalité en soi, que se constituent les représentations et les concepts que l'homme ordinaire se forme du monde, et qui s'imposent à lui $»^{17}$.

15 In PWV, p. 261.

16 Voir notamment le texte de $P W V$ intitulé "Wozu hat der Mensch zwei Augen?», "Pourquoi l'homme a-t-il deux yeux?».

17 Mach E. : L'Analyse des sensations, Nîmes, tr. fr. F. Eggers et J.-M. Monnoyer, Nîmes, J.Chambon, 1992 [ci-après: $A S$ ], p. 33-34. Voir également id., p. 37: «La mission biologique de la science est d'offrir l'orientation la plus complète possible à l'individu humain, doué d'une pleine perception sensorielle. Tout autre idéal scientifique est irréalisable, et dépourvu d'aucun sens». Sur la place que joue cette insistance sur l'origine biologique et physiologique de la science dans l'empirisme de Mach, voir Charrak A., Empirisme et théorie de la connaissance. Réflexion et fondement des sciences au XVIII siècle, Paris, Vrin, 2009, p. 117-118. 
Cette adaptation de la pensée aux faits constitue donc le premier niveau où la notion évolutionniste d'adaptation intervient dans l'épistémologie de Mach. Mais cette notion d'adaptation intervient encore à un second niveau: non seulement lorsqu'il s'agit d'expliquer la relation qui existe entre les pensées et le monde, mais encore lorsqu'il s'agit de comprendre les relations des pensées entre elles. En effet, les pensées elles-mêmes sont soumises à des lois organiques, et il ne suffit donc pas que ces pensées soient adaptées aux faits du monde extérieur pour que la connaissance soit efficace: dans les termes même de Mach, "[l]'adaptation des pensées entre elles est [...] la tâche ultérieure que la pensée doit accomplir en vue de sa pleine satisfaction ${ }^{18}$. C'est pourquoi une pensée authentiquement scientifique sera celle qui remplit pleinement ce double devoir d'adaptation, des pensées aux faits et avec elles-mêmes:

«L'adaptation des pensées aux faits, [...] nous la désignons comme observation, mais l'adaptation des pensées entre elles, nous la désignons comme théorie " ${ }^{19}$.

Cette façon dont Mach fait de la notion d'adaptation la notion cardinale de sa théorie de la connaissance peut du reste le rapprocher de certains auteurs contemporains comme Ludwig Boltzmann qui, eux aussi, développent de façon presque provocatrice ces approches évolutionnistes de la connaissance humaine. Nous citerons ces remarques boltzmaniennes issues de l'article intitulé «Über die Frage nach der objektiven Existenz der Vorgänge in der unbelebten Natur", qui vont jusqu'à faire du cerveau une "machine à produire des images du monde" selon des lois évolutionnistes:

"Quant au cerveau, nous le considérons comme l'appareil, comme l'organe de production des images du monde qui, en raison de la grande utilité de ces images du monde pour ce qui est de la conservation de l'espèce, s'est, conformément à la théorie darwinienne, formé chez l'homme jusqu'à la plus complète perfection, de même que le cou chez la girafe ou que le bec chez la cigogne $»^{20}$.

Pourtant, on constate que, chez Mach, l'adhésion à l'idée d'une épistémologie évolutionniste n'est pas si entière qu'il n'y paraît. En

18 Id., p. 3.

19 Ibid., p. 165.

20 Boltzmann L., «Über die Frage nach der objektiven Existenz der Vorgänge in der unbelebten Natur», in Populäre Schriften, Leipzig, Barth, 1905, p. 179. 
effet, si le physicien reconnaît pleinement la validité de la biologie darwinienne, il n'est nullement évident qu'il entende l'appliquer à la théorie de la connaissance à la simple façon d'un Spencer. Au contraire, on peut être interpelé par ce passage des Leçons scientifiques populaires dans lequel Mach prend manifestement ses distances à l'égard, sinon du contenu, du moins de la démarche qui s'attache aux Principles of Psychologyde Spencer:

"J'entends ici passer sur le thème fort riche de la transmission héréditaire des pensées, ou plutôt de la transmission héréditaire de la disposition à des pensées déterminées. Il ne me conviendrait pas non plus ici de proposer des considérations relatives à l'évolution psychique en général, telles qu'elles ont été exposées avec plus ou moins de bonheur par Spencer ainsi que par de nombreux zoopsychologues modernes ${ }^{21}$.

Une telle prise de distance à l'égard de Spencer est tout à fait frappante, et nous souhaitons ici affirmer qu'elle s'explique notamment par le fait qu'à la démarche strictement descriptive et génétique adoptée par Spencer, Mach entend quant à lui substituer une approche qui associe les idées évolutionnistes à l'élaboration d'une authentique méthodologie ou logique pour la science. C'est là ce qu'il fait en particulier à travers son insistance sur le principe d'économie de pensée, auquel nous allons à présent nous intéresser de plus près.

\section{Le principe d'économie de pensée}

Nous avons déjà eu l'occasion de proposer, dans notre introduction, la formulation canonique du principe machien d'économie de pensée telle qu'on peut la lire dans les pages de la Mécanique. Mais on ne peut en réalité que constater la récurrence de ce principe, qui intervient encore dans bien d'autres textes, notamment dans la conférence de 1882 intitulée "Nature économique de la recherche physique»22. On peut y lire les remarques suivantes:

21 Mach E., Erkenntnis und Irrtum, Skizzen zur Psychologie der Forschung,

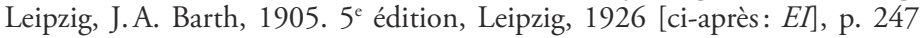
[nous traduisons]. Mach cite ici en note les Principles of Psychology de Spencer.

22 Mach, E., «Die ökonomische Natur der physikalischen Forschung», in PWV. 
«La physique est l'expérience mise en ordre économiquement. Avec cette mise en ordre, on ne rend pas seulement possible une vision d'ensemble de ce qui est déjà acquis: ce sont également les lacunes et les compléments souhaitables que nous traitons ici en bonne économie ${ }^{23}$.

Or c'est justement une telle insistance sur l'exigence économique de mise en ordre des phénomènes ou des connaissances qui permet à Mach, entre autres, de comprendre le rôle que peut jouer la logique proprement dite au sein de la science, et cela parce que les procédures logiques, aussi bien déductives qu'inductives, doivent chez lui être comprises non pas comme des moyens d'étendre nos connaissances, mais de les mettre en ordre. Nous renverrons ici au chapitre 18 de La Connaissance et l'erreur, où Mach écrit que

"syllogisme et induction ne créent donc aucune connaissance nouvelle, mais se contentent d'assurer la production d'absence de contradiction entre nos connaissances, d'exposer clairement leurs connexions, d'attirer notre attention sur différents aspects d'une idée, et de nous apprendre à reconnaître la même idée sous différentes formes " ${ }^{24}$.

Dans ces conditions, il semble effectivement que la logique elle-même, chez Mach, puisse être naturalisée à travers son inscription dans le principe d'économie de pensée et son ancrage évolutionniste. Pourtant, ce sur quoi nous souhaitons insister, pour nous acheminer vers une réinterprétation du principe machien, c'est sur le fait que ce principe d'économie de pensée ne remplit pas seulement une fonction descriptive ou naturaliste: il acquiert en outre, chez cet auteur, une fonction prescriptive. En d'autres termes, ce principe ne permet pas seulement de comprendre en quoi consiste l'activité intellectuelle concrète du scientifique, mais sert également à déterminer les meilleures attitudes à adopter lorsqu'il s'agit de rendre compte des phénomènes sensibles et de les synthétiser dans des lois.

Quelles sont, dans ces conditions, les règles qui sont prescrites au scientifique par le principe d'économie de pensée? Une première remarque sera ici que le seul fait de produire un modèle ou une image des faits remplit déjà une fonction économique, puisque cela nous permet d'enquêter avantageusement sur les représentations plutôt que

23 Op. cit., p. 226.

24 EI, p. 312. 
sur les choses elles-mêmes. De là ces remarques souvent citées de la Mécanique:

"Toute science se propose de remplacer et d'épargner les expériences à l'aide de la copie et de la figuration des faits dans la pensée. Cette copie est en effet plus maniable que l'expérience elle-même et peut, sous bien des rapports, lui être substituée $»^{25}$.

Mais il y a plus: il ne suffit pas, pour respecter le principe d'économie de pensée, d'élaborer des modèles ou des copies des phénomènes sensibles. Il convient de surcroît de les élaborer en respectant un principe de maxi-min, par où l'on cherchera à produire un modèle avantageux des phénomènes qui en sélectionne les aspects saillants tout en en éliminant les détails inessentiels. En ce sens, le principe d'économie de pensée se laisse décomposer en plusieurs sous-principes: un principe de simplicité maximale, un principe de sélectivité des traits pertinents, et une démarche supplétive visant à combler les lacunes de l'expérience. Pour ce qui concerne le premier de ces aspects, Mach insiste sur l'importance du rôle joué par le langage, puisque le seul fait d'embrigader les phénomènes dans un discours, scientifique ou non, opère déjà un travail de schématisation rendu possible par les processus de symbolisation:

"Le langage, moyen de cette communication, est naturellement aussi un facteur d'épargne. Les expériences sont plus ou moins parfaitement décomposées en éléments plus simples et plus familiers, et symbolisées ensuite dans un but de communication, mais toujours en sacrifiant la précision jusqu'à un certain point $»^{26}$.

Or c'est précisément cette règle de simplicité qui, pour pouvoir seulement être observée, exige que l'on procède à une sélection des traits à représenter. Dans les termes de Mach:

«Lorsque nous nous faisons dans la pensée la copie d'un phénomène, celle-ci n'est jamais faite d'après le fait global, mais bien d'après celui de ses côtés qui nous a semblé important. Dans cette opération, nous avons un but qui est le produit indirect ou immédiat d'un intérêt pratique ${ }^{27}$.

Pourtant, le principe d'économie de pensée n'est pas seulement un principe de simplification, de sélection ou d'élimination. Dans certains

$25 M$, p. 449.

26 Id.

27 Ibid., p. 450 [traduction modifiée]. 
cas, au contraire, procéder avec économie signifie ajouter quelque chose à nos modèles, compléter l'expérience, combler les brèches de nos représentations. Mentionnons ici les premières pages de La Connaissance et l'erreur:

«Le but de la vie représentative vulgaire est de compléter ou de parfaire par la pensée un fait partiellement observé. [...] Ce trait qui consiste à compléter par la pensée un fait à partir d'une partie donnée est quelque chose que la pensée scientifique a en commun avec la pensée vulgaire ${ }^{28}$.

Dès lors, si l'on devait rechercher un exemple d'un processus heuristique employé par les sciences et répondant aux règles du principe d'économie de pensée en combinant simplicité, sélectivité et supplémentation de l'expérience, on pourrait tout simplement se référer à la méthode des expériences de pensée, à laquelle Mach consacre notamment un chapitre entier de La Connaissance et l'erreur ${ }^{29}$. En effet, le fait d'expérimenter par la pensée nous permet tout d'abord de faire l'économie du dispositif expérimental, et ainsi, non seulement de nous épargner une dépense matérielle et intellectuelle, mais encore de nous affranchir avantageusement de toutes les contingences liées à ce dispositif, contingences qui pourraient venir fausser les résultats de l'expérimentation: c'est cela que veut dire Mach lorsqu'il écrit, dans le chapitre concerné de La Connaissance et l'erreur, que «[p]ar les pensées, nous expérimentons pour ainsi dire à moindre coût $»^{30}$. Qui plus est, l'expérience de pensée permet de procéder à une simplification de l'expérience et à une sélection de ses traits pertinents, puisqu'elle consiste à élaborer un modèle schématique et simplifié, rendant possible un gain en clarté, mais se constituant également en instance cruciale en vue de décider de la vérité des hypothèses. Enfin, l'expérience de pensée permet bien de compléter l'expérience et d'en combler les lacunes, puisqu'elle

28 EI, p. 2.

29 Mach traite également des expériences de pensée dans d'autres textes, notamment dans la Mécanique, lorsqu'il retrace l'histoire de la science de Galilée ou de Stevin. Voir op. cit., I, 2, p. 35; IV, 4, p. 457. Sur l'attitude générale de Mach à l'égard des expériences de pensée, voir également Plaud S., "Modélisation et expérience de pensée chez Mach: une méthode entre rationalisme et empirisme", in Les Détours du savoir: expérience de pensée, fiction et réalité, Paris, Nouveau Monde, 2009.

30 EI, p. 187. 
rend possible une extension considérable du champ de l'expérience, en nous permettant notamment d'expérimenter là même où une expérience physique eût été impossible. Voilà pourquoi, en définitive, Mach peut caractériser l'expérience de pensée comme "un procédé logicoéconomique de purification en vue de la clarification du contenu de l'expérience mis en forme par la pensée ${ }^{31}$.

\section{L'économie de pensée: une méthode pour la science}

À travers cet exemple de l'expérience de pensée, on s'aperçoit clairement du fait que ces "procédés logico-économiques» qui dérivent du principe d'économie de pensée n’ont pas seulement une portée descriptive, mais bien prescriptive: qu'ils servent effectivement à fonder, fût-ce sur une base naturaliste et évolutionniste, une méthodologie pour la science. Le principe d'économie sert bien ici de critère en vue de déterminer quelles procédures rationnelles ou empiriques sont acceptables, lesquelles ne le sont pas; en vue de déterminer quelles représentations sont acceptables, lesquelles ne le sont pas. Assurément, on a parfois souligné, et à fort juste titre, que l'épistémologie de Mach était une épistémologie naturalisée, qui se confondait presque avec une sorte d'histoire naturelle de la science ${ }^{32}$. Corrélativement, il est tout à fait correct de remarquer que la philosophie machienne de la science et de la connaissance est une philosophie empiriste, qui confère un primat à l'expérience par rapport aux éléments formels de la pensée. Mais ces deux remarques ne doivent pas nous empêcher d'apercevoir la part importante de la pensée de Mach qui est consacrée à l'élaboration d'une méthode pour les sciences naturelles, d'une méthode qui puisse recevoir le statut de logique de la science en tant qu'ensemble de règles formelles, «logicoéconomiques" qui, selon l'expression que nous venons de citer, pourront servir à la "clarification du contenu de l'expérience» en tant qu'il est «mis en forme par la pensée». Cette méthodologie ou cette logique de la science qui est fournie par l'économie de pensée vient ainsi accompagner les analyses biologiques, génétiques, psychologiques ou historiques de

31 Id., p. 188

32 Voir en particulier Garreta G., "Mach: L'épistémologie comme histoire naturelle de la science», in Wagner P. (dir.), Les philosophes et la science, Paris, Gallimard, 2002. 
Mach, et vaut finalement comme l'aboutissement de l'épistémologie de cet auteur.

Pour confirmer cette idée selon laquelle le principe d'économie de pensée, chez Mach, en vient à s'affranchir de ses origines naturalistes pour acquérir une dimension méthodologique et prescriptive, on peut se référer au cas des lois de la nature. Dans l'optique évolutionniste adoptée par Mach, les lois de la nature doivent en effet être réinterprétées comme la manifestation d'une stratégie vitale, comme l'expression d'un «besoin psychologique de nous trouver à notre aise dans la nature, de ne pas rester étrangers ni embarrassés face aux processus $»^{33}$. Mais au-delà de cette explication naturaliste, on constate que Mach, grâce à son principe d'économie de pensée, justifie positivement et sur une base méthodologique cette mise en forme des phénomènes que sont les énoncés nomologiques. Car bien que les phénomènes ne se produisent que de façon unique et singulière (bien que, dans les termes de Mach, la nature ne soit "présente qu'une fois» ${ }^{34}$ ), le scientifique est non seulement autorisé à mais tenu de regrouper ces phénomènes singuliers sous un ensemble de descriptions abrégées, qui en permettront tout à la fois la compréhension et la prédiction:

«Une telle loi de la nature ne contient rien de plus qu'un compte rendu synthétique et condensé des faits. Bien plus, elle contient même moins que les faits eux-mêmes, puisqu'elle ne reproduit pas le fait dans son ensemble, mais uniquement l'aspect de ce dernier qui est important pour nous, tandis que l'on néglige la complétude, intentionnellement ou par besoin. Les lois de la nature sont comparables à des caractères d'imprimerie intellectuels en partie mobiles, en partie stéréotypés, qui peuvent d'ailleurs souvent devenir embarrassants lors de nouvelles éditions de l'expérience $\aleph^{35}$.

Mais il y a plus: dans certains cas, le principe d'économie de pensée peut même prescrire certaines attitudes scientifiques qui prendront le contrepied des tendances naturelles issues de notre évolution biologique: dans de tels cas, il ne fait plus aucun doute que l'épistémologie fondée sur l'économie de pensée s'affranchit de ses origines naturalistes ou génétiques, puisqu'elle nous engage à aller à l'encontre des attitudes

$33 E I$, p. 453-454.

34 Mach E., "Die ökonomische Natur der physikalischen Forschung", in PWV, p. 228.

35 Id., p. 222-223. 
cognitives spontanées telles qu'elles pourraient être décrites par une histoire naturelle de la pensée. Un tel cas de figure peut en particulier être repéré dans le traitement que Mach réserve à un concept qui intervient aussi bien en psychologie qu'en psychophysiologie de la sensation, à savoir le concept de Moi : comme nous aimerions à présent le montrer, ce concept est à la fois susceptible d'être justifié par le principe d'économie de pensée pris dans sa dimension naturaliste et descriptive, et rejeté par ce même principe d'économie, mais cette fois-ci employé dans sa dimension normative et méthodologique. Sur un plan strictement naturaliste, en effet, il serait facile de faire jouer le principe d'économie de pensée dans sa dimension descriptive, pour comprendre quelles tendances biologiques et pragmatiques ont pu conduire les hommes à élaborer une telle notion: l'idée de Moi semble bien comporter une dimension économique, puisqu'elle nous permet de regrouper avantageusement un certain nombre de sensations et de phénomènes formant un complexe relativement doué d'une constance relative. Citons ici L'Analyse des sensations:

«La plus grande fréquence, la suprématie des constantes sur les variables, nous imposent une économie de la représentation et de la dénomination, pour une part instinctive, pour une part volontaire et consciente, qui s'exprime dans la pensée et le langage quotidiens. Ce qui a été représenté de manière unique, reçoit une seule dénomination, un seul nom.

Quant au complexe de souvenirs, d'états d'âme et de sentiments qui se rattachent à un corps particulier (le corps humain), et que l'on désigne en tant que Moi, il se montre relativement stable $»^{36}$.

En ce sens, donc, il semble effectivement que, pris dans sa dimension descriptive (naturaliste, biologique), le principe d'économie puisse servir à expliquer l'émergence du concept de Moi. Mais justement, le propre du principe d'économie chez Mach est de ne pas se limiter à une telle fonction naturaliste, et d'acquérir également une portée normative et méthodologique. Or lorsqu'il assume cette deuxième fonction, ce même principe d'économie de pensée nous montre également les limites que rencontre la notion de Moi, et il engage alors la science naturelle (la psychologie, la psychophysiologie) à s'en affranchir. Tel est le sens très net de cette remarque par où Mach souligne combien les considérations proprement épistémologiques relatives au but de la science doivent

36 AS, p. 8-9. 
parfois s'opposer aux tendances révélées par les approches purement naturalistes de la pensée dans sa fonction pratique:

«Par leur haute signification pratique, non seulement pour l'individu mais pour toute l'espèce, les combinaisons en unités du "Moi" et du "corps" s'affirment de manière instinctive, et se manifestent avec une force élémentaire. Toutefois, dans certains cas particuliers, lorsqu'il ne s'agit pas du but pratique, mais lorsque la connaissance devient une fin de soi, cette délimitation peut se révéler insuffisante, gênante et indéfendable».

Un tel passage est en fait tout à fait remarquable: non seulement parce qu'il permet de ne pas caricaturer le pragmatisme machien en nous montrant comment Mach reconnaît la possibilité relative, pour la science, de s'affranchir de sa finalité pratique première; mais encore, dans l'optique qui nous intéresse, parce qu'il met clairement en lumière la façon dont le principe d'économie de pensée, chez cet auteur, fonde une épistémologie qui va bien au-delà de la simple enquête génétique ou évolutionniste, et qui se constitue en méthodologie normative pour la science, énonçant les principes que doit respecter cette dernière dans son travail de mise en ordre des phénomènes. C'est pourquoi nous conclurons sur cette remarque par laquelle Mach se sert du principe d'économie de pensée pour définir le but ultime, le terminus ad quem de la science:

«Lorsque l'expérience aura clairement exposé ces faits et que la science les aura mis en ordre de façon économique et synoptique, alors il n'y aura pas lieu de douter que nous les comprendrons également. Car il n'y a jamais eu d'autre compréhension qu'une domination du factuel par la pensée » ${ }^{37}$.

\section{Références}

Blackmore J., Ernst Mach, His Work, Life and Influence, Berkeley, University of California Press, 1972.

Boltzmann L., Populäre Schriften, Leipzig, J. A. Barth, 1905.

37 Mach E., «Die ökonomische Natur der physikalischen Forschung», in PWV, p. 239-240. 
Bouveresse J., «Hertz, Boltzmann et le problème de la "vérité» des théories", in Lichnérowicz A. et Gadoffre G. (dir.), La vérité estelle scientifique?, Paris, Éditions universitaires, 1991.

Garreta G., "Mach: L'épistémologie comme histoire naturelle de la science, in Wagner P. (dir.), Les philosophes et la science, Paris, Gallimard, 2002.

Lahbib O., "Économie de pensée et Énergétique chez Ernst Mach», in Ghesquier-Pourcin D., Guedj M. et Goahau G. (dir.), Énergie, science et philosophie au tournant des XIXe et XX siècles, vol. 2: Les Formes de l'énergétisme et leur influence sur la pensée, Paris, Hermann, 2010, p. 99-111.

Lahbib O., "La signification vitaliste de la connaissance chez Ernst Mach», document web, site «Dogma», http://www.dogma.lu/ txt/OLahbib-Mach.htm.

Lahbib O., «Ernst Mach : une théorie de la science non-transcendantale», document web, http://mondodomani.org/dialegesthai/ol01. htm\#par2.

Mach E., Beiträge zur Analyse der Empfindungen, Prague, 1886. Traduction française par F. Eggers et J.-M. Monnoyer: L'Analyse des sensations, Nîmes, J. Chambon, 1992.

Mach E., Erkenntnis und Irrtum, Skizzen zur Psychologie der Forschung, Leipzig, J. A. Barth, 1905. $5^{\mathrm{e}}$ édition, Leipzig, J.A. Barth, 1926. Traduction française par M. Dufour: La Connaissance et l'erreur, Paris, Flammarion, 1908.

Mach E., "Facts and Mental Symbols", in The Monist, janvier 1892, p. 198-208.

Mach E., Die Geschichte und Wurzel des Satzes von der Erhaltung der Arbeit. Vortrag gehalten in der k. böhm. Gesellschaft der Wissenschaften am 15. November $1871.1^{\mathrm{e}}$ edition, Prague, 1872. Traduction anglaise par Ph.E. B. Jourdain: History and Roots of the Principle of the Conservation of Energy, Chicago, Open Court, 1911.

Mach E., Die Mechanik in ihrer Entwicklung, historisch-kritisch dargestellt, Leipzig, 1904, 1883. Traduction française par É. Bertrand: La Mécanique, Exposé historique et critique de son développement, Paris, Hermann, 1904, reproduit chez J. Gabay, Sceaux, 1987.

Mach E., Populär-wissenschaftliche Vorlesungen, $3^{\mathrm{e}}$ édition, Leipzig, J.A. Barth, 1903.

Mach E., Die Principien der Wärmelehre, Historisch-kritisch entwickelt, Leipzig, J.A. Barth, $2^{\mathrm{e}}$ édition, 1900. 
Plaud S., "L'épistémologie aux origines de la philosophie analytique», in Laugier S. et Plaud S., Lectures de la philosophie analytique, Paris, Ellipses, 2011.

Plaud S., "Modélisation et expérience de pensée chez Mach: une méthode entre rationalisme et empirisme", in Les détours du savoir: expérience de pensée, fiction et réalité, Paris, Nouveau Monde, 2009.

Regnaud P., Précis de logique évolutionniste, Paris, Alcan, 1897.

Spencer H., The Principles of Psychology, Londres, Longman, Brown, Green and Longmans, 1855; la pagination mentionnée dans les citations de cet article correspond à la version numérique du texte disponible sur le site Libertyfund.org: http://files.libertyfund. org/files/1394/0625_Bk.pdf. 